Gianfranco Fiaccadori

Università degli Studi di Milano

\title{
UN RE DI NUBIA A COSTANTINOPOLI NEL 1203*
}

Robert de Clari, celebre cronista francese della IV Crociata, nell'agosto 1203 incontrò alla corte di Costantinopoli «uns rois qui toute avoit le char noir, et avoit une crois en mi le front qui li avoit esté faite d'un caut fer». ${ }^{1}$ L'imperatore Alessio IV Angelo così presentava il monarca ai baroni franchi dai quali era stato appena rimesso sul trono: «ch'est li rois de Nubie, qui est venu en pelerinage en cheste vile»; poi, a mezzo d'interpreti (latimiers), l'ospite fu interrogato dai presenti sulla sua terra d'origine: «Et fist on parler latimiers a lui, et fist on lui demander ou se tere estoit, tant qu'il respondi as latimiers, en sen langage». ${ }^{2}$ Il «rois de Nubie», cui già Alessio III (avanti il 17/18 luglio di quell'anno) aveva dato in uso un monastero della città («une molt rike abeïe en le chité»), dichiarò allora d'esser partito dal suo Paese, «encore cent journees dela Jherusalem», con un séguito di sessanta uomini: a Gerusalemme ne rimanevano dieci, a Costantinopoli due solamente, coi quali egli intendeva recarsi «en pelerinage a Rome et de Rome a Saint Jake [di Compostella, in Galizia] et puis revenir s'ent ariere en Jherusalem, s'il pooit tant vivre, et puis illueques morir. Et si dist que tot chil de se tere estoient crestien, et quant li enfes estoit nes et on le baptisoit, que on li faisoit une crois en mi le front d'un caut fer ausi comme il avoit». ${ }^{3}$

A Benjamin Hendrickx si deve la più recente e singolare proposta circa l'identità dell' anonimo sovrano: si tratterebbe del nəguś Lalibäla (c. 1185-1225), «costruttore» delle celebri chiese monolitiche nella città che da lui prende nome

${ }^{*} C E=$ The Coptic Encyclopaedia, ed. by Aziz S. Atiya, New York, \&c., Macmillan Publishing Co., \&c., 1991, voll. 1-8; ODB = The Oxford Dictionary of Byzantium, ed. by A. Kazhdan \& al., New York — Oxford, O.U.P., 1991, voll. 1-3.

${ }^{1}$ Robert de Clari, La Conquête de Constantinople, LIV, éd. par Ph. Lauer, Paris, E. Champion, 1924 (Les classiques françaises du Moyen Age, 40), 54.4-55.1 [= Historiens et chroniquers du Moyen Age, éd. établie et annotée par A. Pauphilet, textes nouveaux commentés par E. Pognon, Paris, Gallimard, 1952 (Bibliothèque de la Pléiade, 48), 13-91: 54.25-27]. Cf. M. McC(оRMICK), «Robert de Clari», in: $O D B$ vol. 3, 1799, con bibliogr., cui ora si aggiunga P. ScHreINER, Robert de Clari und Konstantinopel, in: Novum Millennium. Studies in Byzantine history dedicated to Paul Speck 19 Dec. 1999, ed. by C. Sode - S. Takács, Aldershot, \&c., Ashgate, 2001, 337-356.

${ }^{2}$ Robert de Clari, La Conquête de Constantinople, 1. cit., 55.14-18 [= Historiens et chroniquers du Moyen Age, 54.34-38].

${ }^{3}$ Ivi, 55.6-7 e $18-28$ [= 54.27-28 e 54.36-55.3]. 
fra i monti del Lasta, in Etiopia. ${ }^{4}$ Tale proposta, accolta nell' Oxford Dictionary of Byzantium, ${ }^{5}$ non va però immune da dubbî e incertezze, priva com'è di riscontri, se non leggendarî, da parte abissina: che Lalibäla fosse miracolosamente trasportato a Gerusalemme per compiere il pellegrinaggio ai Luoghi Santi si apprende infatti dal solo suo $G \ddot{a} d l$ ( $B H O$ 560), la «Vita» composta verso la metà del XV secolo. ${ }^{6}$ Del resto, Hendrickx, il cui lavoro è sfuggito alla recente sintesi di Derek A. Welsby sulla Nubia medievale, non conosce la precedente interpretatio Nubiana di Bożena Rostkowska, che nell'ospite di Alessio IV ha invece scorto «a king of Makuria or one of the lesser Nubian rulers». ${ }^{7}$

${ }^{4}$ B. HENDRICKX, «Un roi africain à Constantinople en $1203 », B \nu \zeta \alpha \nu \tau \iota \nu \alpha ́ 13,1985$, 893-898.

${ }^{5}$ Vd. D.W. J(OHNSON) - A. K(AZHDAN), «Nubia», in: $O D B$ vol. 3, $1500 a-b$.

${ }^{6}$ Questa parte, omessa nella Vie de Lalibala, roi d'Éthiopie, \&c., publiée par J. Perruchon, Paris, E. Leroux, 1892, 37.8 (t.) = 103.19 (tr.), può leggersi nei c.d. Atti di Mäsqäl Kəbra, pubblicati da S. KuR, «Édition d'un manuscrit éthiopien de la Bibliothèque Vaticane: Cerulli 178», MRALm, sc. mor., ser. VIII, 16 (7), 1972, 383-425: 387.12-402.6 (t.) = 406.24-421.5 (tr.). Vd. ora la descriz. sommaria del cod. (sec. XIX) in E. Cerulli, Inventario dei manoscritti Cerulli etiopici, Introduzione, integrazioni e indici a c. di O. Raineri, Città del Vaticano, B. A. V., 2004 (Studi e testi, 420), 130 s. Cf. Kinefe-Rigb ZelleKe, «Bibliography of the Ethiopic Hagiographical Traditions», JES 13/2, 1975, 57-102: 79 s., nr. 89; R. SAUTER, «Lalibäla», in: DEB 101 s.; e sopratutto S. TedeschI, «Lālibalā», in: Bibliotheca Sanctorum orientalium (Enciclopedia dei Santi. Le Chiese orientali), II, Roma, Città Nuova Ed., 1999, 300303. Per il nesso con Gerusalemme: J. Pirenne, La légende du «Prêtre Jean», Strasbourg, Presses universitaires, 1992, 44 ss.; M. E. Heldman, «Architectural symbolism, sacred geography and the Ethiopian Church», Journal of Religion in Africa 22, 1992, 222-241; EAD., «Legends of Lālibalā. The development of an Ethiopian pilgrimage site», Res 27, 1995, 25-38. Ma vd. ora E. vAN DonZEL, «Lālibalā and Jerusalem in the Twelfth Century», in: East and West in the Crusader States: context - contacts - confrontations, [I]. Acta of the congress held at Hernen Castle in May 1993, ed. by K. Ciggaar \& al., Leuven, Uitgev. Peeters, 1996 (OLA, 75), 73-80; ID., «Were there Ethiopians in Jerusalem at the Time of Saladin's Conquest in 1187?», ivi, II. Acta of the congress held at Hernen Castle in May 1997, ed. by K. Ciggaar \& H. Teule, Leuven, Uitgev. Peeters, 1999 (OLA, 92), 125-130; ID., «Ethiopia's Lalibäla and the fall of Je-rusalem 1187», Aethiopica. International Journal of Ethiopian Studies 1, 1998, 27-49.

${ }^{7}$ Vd. risp. D. A. Welsby, The Medieval Kingdoms of Nubia: Pagans, Christians and Muslims along the Middle Nile, London, The British Museum P., 2002, 76 e n. 47 (265), e B. Rostkowska, «The visit of a Nubian king to Constantinople in A.D. 1203», in: New Discoveries in Nubia, Proceedings of the Colloquium on Nubian Studies. The Hague, 1979, ed. by P. Van Moorsel, Leiden, Nederlands Instituut voor het Nabije Oosten, 1982 (Egyptologische Uitgaven, II), 113-116. Incerto fra Nubia ed Etiopia/Abissinia G. VAntini, Il Cristianesimo nella Nubia antica, Verona, Novastampa, 1985 (Museum Combonianum, 39), 234: «...però la possibilità che si tratti proprio di un re nubiano non si può escludere $a$ priori». 
Ora, benché gli Etiopi siano effettivamente associati ai Nubiani e spesso confusi con questi nelle fonti antiche e medievali, ${ }^{8}$ è da credere che li distinguesse la cancelleria bizantina; e più tardi, fra il 1252 e il 1295, l'interpolatore (un monaco di Huy, presso Liegi) della cronaca di Alberico delle Tre Fontane segnala presbiteri nubiani e, necessariamente, abissini (sudditi del Prete Gianni) officianti al Santo Sepolcro: «...Septimus ordo dicitur Nubianorum Christianorum, quorum terra maxima est et multi ex eis tributarii Sarracenorum sunt. Octavus ordo totam illam multitudinem Christianorum continet que Presbitero Ioanni subiecta est». ${ }^{9}$ Contrariamente a quanto ritiene Hendrickx, ${ }^{10}$ non sembra poi decisiva l'indicazione di Robert de Clari sulle «focature» battesimali, attributo canonico degli uni e degli altri: ad esempio, nel dipinto della Cattedrale di Faras (l'antico vescovado di Pachōras, città regia di Nobadia) la «principessa» nubiana protetta dalla Vergine col Bambino mostra precisamente «une crois en mi le front» (il pannello, dell'XI/XII secolo, è oggi al Sudan National Museum di Huartūm). ${ }^{11}$ E così potrà intendersi, per Gerusalemme, la testimonianza oculare del domenicano Burcardo da Monte Sion, circa il 1280-1285: «...Nubeani \& Jabeani [= Abissini] dicunt se scire, majores suos fidem suscepisse, quam hodie inviolabiliter servare contendunt, innitentes huic authoritati, Matth. III:11. Ipse vos baptizabit in Spiritu Sancto

${ }^{8}$ Vd., p. es., G. F., Ethiopians, in: $O D B$ vol. 2, $733 a-b$, con bibliogr.; ID., Teofilo Indiano, Presentazione di R. Farioli Campanati, Ravenna, Ed. del Girasole, 1992 (Biblioteca di «Felix Ravenna», 7), 12 ss., n. 33, 46 s., n. 12, con altre indicaz.; Ph. MAYerson, «A confusion of Indias: Asian India and African India in the Byzantine sources», Journal of the American Oriental Society 113, 1993, pp. 169-174 (= ID., Monks, Martyrs, Soldiers and Saracens. Papers on the Near East in Late Antiquity, Jerusalem, The Israel Explor. Soc. in collab. with N. Y. Univ., 1994, 361366), di cui R. Contini, Quaderni di studi arabi 12, 1994 [ma 1995], 217-221: 220.

${ }^{9}$ Chronica Albrici monachi Trium Fontium, A. 1234, ed. P. Scheffer-Boichorst, in: Monumenta Germaniae historica. Scriptores, XXIII, Hannoverae, Imp. Bibliopolii Aulici Hahniani, 1874, 631-950: 935.35-39, discusso e riprod. da E. CERULLI, Etiopi in Palestina. Storia della comunità etiopica di Gerusalemme, I, Roma, La Libreria dello Stato, 1943 (Collezione scientifica e documentaria a c. del Ministero dell'Africa Italiana, XII), 77 ss.: 79, doc. nr. 9. L' «octavus ordo» è impropriamente riferito alla Nubia in VANTini, Il Cristianesimo, 235. Sul Prete Gianni vd. infra e n. 19.

10 «Un roi africain à Constantinople», 897 n. 14.

${ }^{11}$ Rostkowska, «The visit of a Nubian king», 116 e n. 12, pl. II, fig. 2. Cf. E. DinKLER, «Beobachtungen zur Ikonographie des Kreuzes in der nubischen Kunst», in: Nubia. Récentes recherches, Actes du Colloque nubiologique international au Musée de Varsovie (19-22 juin 1972), sous la réd. de K. Michałowski, Varsovie, Musée National, 1975, 22-30: 27 s. e nn. 36-37, fig. 7. Sul segno della croce, «impresso con antimonio, indelebile» per VANTINI, 1. cit., vd. sopratutto E. CERULLI, «Il volo di Astolfo sull'Etiopia nell' Orlando Furioso», RRALm, sc. mor., ser. VI, 8, 1932, 19-38: 28-33, e ID., Etiopi in Palestina, I, 51 ss., 58 s. 
\& igne. Nam cuum baptizantur, cum calido ferro crucem inurunt faciei \& alteri corporis parti. Et hi devotiores inter Orientales habentur». ${ }^{12}$ Alla medesima altezza cronologica del testo di Burcardo, «Abasce» e «Nubie» sono chiaramente individuate e distinte nel Milione di Marco Polo, con «une bielle estoire que avint a les MCCLXXXVIII anz de l'a[n]carnasionz de Cristi»: il «roi (de la provence) de Abasce» o «d'Abasce» — dall' arabo Habašah 'Abissinia' - è in stato di guerra «con le soudan de Aden et con celz de Nubie»; vuol recarsi pellegrino al Santo Sepolcro («por aorer le sepolcre de Crist en Jerusalem») e manda però in vece sua un vescovo poi catturato sulla via del ritorno e circonciso dal «soudan de Aden», ossia da un capo dei Begia (Buğah) della regione nota agli itinerarî quattrocenteschi come Adam o $A d(d) a n$, fra il delta del Barca (Barka) e il confine eritreo (e ciò vale a restituire i fatti al tempo del nəguś Yagbəa Șəyon, 1285-1294). ${ }^{13}$

Ritengo pertanto che la testimonianza di Robert de Clari debba riferirsi a un re o a un principe nubiano in transito o in cerca d'appoggi contro gli Ayyūbidi di Saladino (Ṣalāḥ ad-Dīn), l'avvento dei quali, nel 1172, avrebbe del

${ }^{12}$ Descr. Terrae Sanctae, [XIII 1], ed. H. Canisius, Thesaurus monumentorum ecclesiasticorum et historicorum, sive Lectiones antiqua, \&c., IV, Antverpiae, ap. R. \& G. Wetsenios, 1725, 9-26: 24.15-19, discusso e riprod. da Cerulli, Etiopi in Palestina, I, 80-84: 84, doc. nr. 10. Il passo manca nelle redazioni fatte conoscere da J. C. M. Laurent, Peregrinatores medii aevi quattuor, Lipsiae, J. C. Hinrichs bibliopola, 1864, 1-100: 89, e H. OMONT, «Manuscrits de la Bibliothèque de Sir Thomas Phillips récemment acquis pour la Bibliothèque Nationale», Bibliothèque de l'École de chartes 64, 1903, 490-553: 498-503 (500).

${ }^{13}$ Marco Polo, Il Milione, CXCIV.17-85, Prima ed. integrale a c. di L. F. Benedetto, Firenze, L. S. Olschki-Ed., 1928 (Comitato Geografico Naz. Italiano-Pubblicaz., 3), 210 s. = Milione, [189], 1-13, Vers. toscana del Trecento. Ed. critica a c. di V. Bartolucci Pizzorusso. Indice ragionato di G. R. Cardona, Milano, Adelphi, 1975 (Classici, 31), 293 s. A rettifica di C. Contr Rossini, «Marco Polo e l'Etiopia», Atti del Reale Istituto Veneto di scienze, lettere ed arti 99, Parte II, 1939-1940, 10211039: 1027, che pensava all'arabo ${ }^{c}$ Adal (etiop. Adäl), la «regione musulmana ad est dello Scioa, nel bassopiano», vd. E. CERULLI, «Il Sultanato dello Scioa nel secolo XIII secondo un nuovo documento storico», RSE 1, 1941, 5-42: 19 n. 1 (= ID., L'Islam di ieri e di oggi, Roma, I.P.O., 1979 [Pubblicazioni, 64], 207-243: 222 n. 68), e ID., Etiopi in Palestina, I, 109 ss.: 109 e n. 2, ma sopratutto S. TEDESCHI, «L'Abissinia nel Libro di Marco Polo», Africa (Roma) 36, 1981, 361-389: 383-386 (viaggi del fiorentino Antonio Bartoli e del messinese Pietro Rombulo), sfuggito a S. C. MunroHAY, Ethiopia and Alexandria. The Metropolitan Episcopacy of Ethiopia, Mit einem Vorwort v. M. Kropp, Warszawa - Wiesbaden, Zaś Pan, 1997 (Bibliotheca Nubica \& Aethiopica, 5), pp. 195, 203; nonché a E. WAGNER, «Adal», in: EAE I, 71a-72b: $72 a$, e D. Morin, Dictionnaire historique afar (1288-1982), Paris, Karthala, 2004 (Collection «Hommes et Sociétés», s. n.), 32-35, s. v. «Adal»: 33, ove la «bielle estoire» di Polo è datata tout court al 1295. 
tutto isolato la Nubia dalle comunicazioni col Mediterraneo..$^{14}$ Né può escludersi una certa solidarietà con gli ultimi Fāțimidi che, rifugiatisi in territorio nubiano, da qui istigavano alla rivolta contro l'Egitto. ${ }^{15} \mathrm{E}$ poco importa che Costantinopoli fosse allora «under the control of the Crusaders», ${ }^{16}$ poiché interlocutori del grecofono «rois de Nubie» erano manifestamente i Bizantini, presso i quali egli già dimorava al tempo di Alessio III (ma certo un'allenza fra Nubiani e Crociati sembra profilarsi dopo il 1274, in esito al Concilio di Lione).$^{17}$ La pagina del cronista dà infatti luce, fra Gerusalemme e Costantinopoli, su contatti e relazioni dai quali fu alimentato nei secoli, ben oltre l'impulso iniziale, il peculiare Graecismus dei regni cristiani di Nubia. ${ }^{18}$ Un Graecismus assai meno conservativo di quanto non si ritenga per solito; e forse non del tutto estraneo all'inserzione (naturalmente polemica) di Manuele I Comneno fra i destinatarî della famosa lettera del Prete Gianni, che l'arcive-

${ }^{14}$ W. Y. Adams, Nubia, Corridor to Africa, Princeton, NJ, P.U.P., 1977, 456 (739); Vantini, Il Cristianesimo, 276. Cf. S. Hillelson - (C. E. Bosworth), «Nūba, 2. History», in: The Encyclopaedia of Islam, ed. by C. E. Bosworth \& al., VIII/1 (131), Leiden, E. J. Brill, 1993, 90a-91b: 90a-b.

${ }^{15}$ U. Monneret de Villard, Storia della Nubia cristiana, Roma, P.I.O.S., 1938 (OCA, 118), 197 s. (cf. 225: inesatto); Welsby, The Medieval Kingdoms, 75 ss. (265).

${ }^{16}$ Così Welsby, The Medieval Kingdoms, 76 s. e n. 48 (265), che rinvia a J. W. CRAwFoot, «Christian Nubia», Journal of Egyptian Archaeology 13, 1927, 141 150: 148 s., ma ignora Cerulli, Etiopi in Palestina, I, 140 s., 143 s. per la cappella nubiana del Golgota o di Adamo, e O. F. A. MeInARdus, «The Christian Kingdoms of Nubia», Cahiers d'histoire égyptienne 10, 1967, 133-164: 159-164 («The Nubians in the Holy Places»), nonché, più in generale, A.-D. v. DEN BRINCKEN, Die «Nationes Christianorum Orientalium» im Verständnis der lateinische Historiographie von der Mitte der 12. bis in die zweite Hälfte des 14. Jahrhunderts, Köln — Wien, Böhlau Verlag, 1973 (Kölner historische Abhandlungen, 22), 243-262 (4. «Die Nubier»); cf. RostKowsKa, «The visit of a Nubian king», 114 n. 5.

${ }^{17}$ Vd. supra e n. 3. Per il cenno al Concilio di Lione, G. Vantini, «Sur l'éventualité des rapports entre le Concile de Lyon (1274) et la Nubie», Études nubiennes, Colloque de Chantilly (2-6 juillet 1975), Le Caire, I.F.A.O., 1978 (Bibliothèque d'étude, LXXVIII), 337-345, e ID., Il Cristianesimo, 243.

18 Vd. T. HäGG, «Greek Language in Christian Nubia», in: $C E$ vol. 4, $1170 b-$ $1174 a$, con bibliogr.; e ora ID., «Greek in Upper Nubia: An Assessment of the New Material», in: Actes de la VIIIe Conférence internationale des Études nubiennes (Lille 11-17 septembre 1994), Lille, Université Charles-De-Gaulle — Lille III, 1998 (Cahiers de recherches de l'Institut del Papyrologie et d'Egyptologie de Lille, 17), III. Etudes, 113-119: 114 ss., e WelsBy, The Medieval Kingdoms, 236 ss. (272). Inoltre, A. ŁaJTAR, Catalogue of the Greek Inscriptions in the Sudan National Museum at Khartoum (I. Khartoum Greek), Photographs by W. Godlewski, Preface by Hassan Hissein \& W. Godlewski, Leuven - Paris - Dudley, MA, Uitgev. Peeters, 2003 (OLA, 122), XIX s. e n. 9, e già ID., «Greek Funerary Inscriptions from Old Dongola: General Note», OC 81, 1997, 107-126: 115 s. 
scovo Cristiano di Magonza avrebbe portato con sé da Costantinopoli nel 1170: ma anzi congeniale al relais «bizantino» della leggenda intorno a quel favoloso monarca, divenuto per tempo «rex et sacerdos» d'un remoto paese africano (com'è già per l'interpolatore di Alberico delle Tre Fontane). ${ }^{19}$

Determinante per l'identità etnica del «rois de Nubie» è senz'altro la circostanza che egli si esprimeva «en sen langage»: vale a dire in greco, l'antica lingua di cultura ancor viva nel suo regno, come dimostra la continuità d'uso nei papiri, pergamene ed epigrafi d'area nubiana. ${ }^{20}$ Per questo egli poté comunicare con il cronista e gli altri baroni franchi a mezzo di latimiers o 'interpreti': dal greco, non certo dal nubiano o dall'etiopico, la cui conoscenza stupirebbe davvero nella Bisanzio dell'epoca.

Quanto alla precisa localizzazione del suo dominio, si può naturalmente pensare al regno di Macuria (Muqurrah) o Dongola (Dunqulah, dal nome della sua capitale): la «Grande Nubia», in parte corrispondente all'odierno Sudan e islamizzata solo dal 1317; il suo sovrano è «re di Nubia» per eccellenza anche presso gli autori di lingua araba. ${ }^{21}$ Ma non escluderei il regno meridionale di 'Alwah o 'Alwā (l'antica Alōdia, Alōa), con capitale Soba/ Sūbah (la Soba Noba della tradizione abissina), ${ }^{22}$ fiorente «corridor to Africa» governato allora da prìncipi cristiani ${ }^{23}$ o uno dei piccoli Stati settentrionali vassalli della «Grande Nubia», ugualmente improntati di cultura bizanti-

19 Testo della lettera in F. ZARnCKE, «Der Priester Johannes», I., Abhandlungen der philol.-hist. Classe der Kgl. sächsischen Gesellschaft der Wissenschaften 7, 1879, 827-1039: 909-924 [= E. UllendorfF - C. F. Beckingham, The Hebrew Letters of Prester John, Oxford, O.U.P., 1982, 184-189]; vd. ora La lettera del Prete Gianni, a c. di G. Zaganelli, Parma, Pratiche Ed., $1990^{2}$ (Biblioteca medievale, 13), 52-95 = Milano - Trento, Luni Ed., 2000 (Biblioteca medievale, 73), 52-95 per la vers. latina, con ampie indicaz. e bibliogr. nell'Introduzione, 7-44 =7-42, e nella Nota informativa, 45 s. = 43 s. Cf. E. Cerulli, «Prester John», in: DEB 133 s., e già ID., «Prete Gianni», in: Enciclopedia Italiana, XVIII, Roma, I. E. I., 1935, 216f-218a; altre indicaz. in A. PonTANI, «Paralipomeni dei Turcica: gli scritti di Giano Lascaris per la crociata contro i Turchi», Römische historische Mitteilungen 27, 1985, $213-$ 338: 324 ss. Inoltre, PIRENne, La légende du «Prêtre Jean», 83-86 («La Lettre à l'Empereur de Byzance»), cui si oppone I. BeJczy, La Lettre du Prêtre Jean, une utopie médiévale, Paris, Imago, 2001, 13-83 in partic. (non convincente), con la rec. di M.-L. Derat, Aethiopica. International Journal of Ethiopian Studies 5, 2002, 239 242: 241 s., dei quali E. UllendorfF, ivi, 7, 2004, 288.

${ }^{20}$ Cf. supra, n. 18: in partic., HäGG, «Greek Language in Christian Nubia», 1172 ss. (1173 s.), e Welsby, The Medieval Kingdoms, 238.

${ }^{21}$ Welsby, The Medieval Kingdoms, 92 s., 251; cf. Monneret de Villard, Storia, 172 ss.

${ }^{22}$ C. Contr Rossini, «Piccoli studi etiopici», Zeitschrift für Assyriologie 27, 1912 , 358-378: 365 ss., con le indicaz. nel mio Teofilo Indiano, 17 s., n. 52.

${ }^{23} \mathrm{Vd}$. Monneret de Villard, Storia, 147-157; Adams, Nubia, Corridor to Africa, 536-539 (753); Welsby, The Medieval Kingdoms, 254 s. (273) in partic., con la 
na: ${ }^{24}$ forse quel misterioso Dotawo - sito nel Marīs o Nobadia (la Bassa Nubia, provincia settentrionale di Macuria) e comunque sovrapponibile a questa - che esisteva fin dal XII secolo intorno al Ğabal ' Addā ( $a$ d - Daww dei mss. arabi medievali), c. $55 \mathrm{~km}$ a sud di Qașr Ibrīm (la romana Primis Parva). ${ }^{25}$ È lo Stato nubiano che rimase più a lungo cristiano, in relativa autonomia dal suo «parent kingdom» e ben oltre la rovina di questo, di cui assunse anzi il ruolo dal 1365/6 in séguito all'abbandono dell'antica Dongola, spegnendosi poi lentamente verso il 1500. ${ }^{26}$ I documenti nubiani di Qașr Ibrīm, che assicurano circa l'importanza del Dotawo, ne conoscono un re, Bas(i)l(ios), asceso prima del $1199 \mathrm{e}$ ancora sul trono nel 1200:27 ma è prudente resistere alla tentazione di scorgere in lui, o nell'ignoto suo successore, il «rois de Nubie» incontrato a Bisanzio da Robert de Clari.

\section{SUMMARY}

In A. D. 1204, a «king of Nubia» who had been a pilgrim to Jerusalem was met by the French chronicler Robert de Clari at the court of Constantinople. Here the former was questioned, through interpreters, by the Frankish barons then gathering around the newly restored Emperor Alexios IV. That king has been lastly identified with the Ethiopian nəguś Lalibäla (r. 1185-1225 ca.), whose legendary relationships with Jerusalem are well known. Such a doubtful identification has even crept into a standard reference work, the Oxford Dictionary of Byzantium. Yet, in accordance to previous scholarship, the king in question is more likely to have been a Greek-speaker Nubian ruler of either the kingdom of Alwa ( $\left.{ }^{\mathrm{C}} \mathrm{Alwah}\right)$ or the kingdom of Makuria (Muqurrah) — or rather of one of the latter's vassal states, possibly Dotawo in Lower Nubia.

rec. di S. DONADONI, Orientalia n.s. 73, 2004, 270-273: 273, e già E. ZyHLARZ, «I reami della Nubia prima dell'Islam: uno sguardo storico sul Sudan antico e medievale», RSE 3, 1943, 237-271: 268 ss.

${ }^{24} \mathrm{Cf}$. RostKowska, «The visit of a Nubian king», 115 e n. 9.

${ }^{25}$ Monneret de Villard, Storia, 140 ss.; Adams, Nubia, Corridor to Africa, 466 s. (741), 531-536, 542 (752); ID., «Dotawo», in: $C E$ vol. 3, 922b-923a; «Jabal 'Addā», ivi, vol. 4, 1315a-b; ID., "Qașr Ibrīm», ivi, vol. 7, 2036b-38a; WelsBy, The Medieval Kingdoms, 250-254 (273).

${ }^{26}$ J. M. Plumley, «The Christian Period at Qasr Ibrim. Notes on the MS finds», in: Nubia. Récentes recherches, 101-107: 105 s.; ID., «Qasr Ibrim and Islam», Etudes et travaux (Warsaw) 12, 1983, 157-170: 164 ss.; VANTINI, Il Cristianesimo, 272 ss.; Welsby, The Medieval Kingdoms, 251 e 254, con terminus post quem agli anni ' 60 del sec. XVI: «The kingdom of Dotawo seems to have vanished in the intervening period».

${ }^{27}$ G. M. Browne, Old Nubian Texts from Qașr Ibrīm, III, London, Egypt Exploration Society, 1991, 14 (t.) = 54 (tr.), nr. 37.3-4 (26 feb., s.a.); $16=56$, nr. 38.5-7 (30 dic. 1199); $18=58$, nr. 39.5-7 (27 giu., s. a.); $20=59$, nr. 40.3 (16 ag. 1200). Cf. J. M. Plumley, «New Light on the Kingdom of Dotawo», in: Études nubiennes, 231-242: 234, 239 s. 